\title{
Pervasive Gaming as a Potential Solution to Traffic Congestion: New Challenges Regarding Ethics, Privacy and Trust
}

\author{
Vincent Koenig ${ }^{1,2}$, Franziska Boehm ${ }^{2}$, and Rod McCall ${ }^{2}$ \\ ${ }^{1}$ EMACS Research Unit \& \\ ${ }^{2}$ Interdisciplinary Centre for Security, Reliability and Trust \\ University of Luxembourg \\ Luxembourg-Kirchberg, L-1359 \\ \{Vincent.koenig, franziska.boehm, roderick.mccall\}@uni.lu
}

\begin{abstract}
The following paper presents a review of the ethical, privacy and trust aspects relating to pervasive gaming in particular within the domain of traffic congestion. The paper deals explicitly with the challenges involved that fall between the gaps standard ethical practice and scientific research when studies comprise of those in the lab (where collection and use is heavily controlled) and those which take place in the wild where there is the requirement to share data possibly with external parties. Also where the nature of such work is at the borders of the concept of traditional study and a commercial running prototype.
\end{abstract}

Keywords: ethics, privacy, trust, game, car, traffic.

\section{Introduction}

The I-GEAR project (incentives and gaming environments for automobile routing) aims to understand the motivations that drivers have while undertaking their daily commute and then to provide them with a range of incentives to change their behaviour with the aim of reducing traffic congestion. A key aspect of the project is on ways in which the problem could potentially be solved without recourse to expensive roadside infrastructure. As a result our solution involves encouraging commuters to use their mobile devices within a serious pervasive (and persuasive) gaming context.

While technical and human-computer interaction aspects are highly relevant within this project a number of the challenges lie outside these areas. For example there is a complex interplay between the technical, human, legal and ethical issues that need to be dealt with. This paper illustrates how the I-GEAR project triggers questions pertaining to ethics, privacy and trust in the context of a serious game that is meant to reduce traffic within Luxembourg. Furthermore, while there are no doubt issues relating purely to Luxembourg the higher level ethical, privacy and trust issues should also be applicable in other countries. We start by outlining the main ideas behind our game model and describe in the second stage specific questions regarding ethics, 
privacy and trust. It is important to note that this is a sample game design concept and the final design will depend on early user requirements modelling. Additionally, rather than providing out-of-the-box answers, we discuss these issues under the light of specific expertise, that we believe goes someway beyond the traditional interdisciplinary approaches within IT research. Ultimately, we would like this paper to foster awareness for the importance of ethics and legal frameworks to be taken into account in pervasive gaming.

\section{I-Gear: Sample Game Model}

The game we intend to develop will run on smartphones for two reasons: firstly, the number of smartphones sold to adults has expanded significantly in recent years (thereby creating a ready potential user base) and secondly, the vast majority of smartphones come with localization (GPS most of the time) and data networking capabilities. Furthermore, in contrast to systems integrated into the cars, this allows us to build on the most recent and widespread technology, to easily bind the device to a specific user, to easily deploy updates and to have easy and instant access to a large number of potential users.

I-GEAR relies on the concept of incentives being provided to drivers within a game-like environment. Incentives have already been used within traffic management and studies have found that even for a comparatively low cost people will alter their driving behaviour [1]. I-GEAR does not claim to be able to remove costs completely, but instead aims to minimize costs by providing financial and non-financial incentives to encourage drivers to change their behaviour. I-GEAR will develop a pervasive game in the sense that it attempts to alter (or persuade) drivers to change their behaviour through the use of social, psychological, financial or game design incentives. Drivers for example may undertake individual actions such as: giving other drivers priority, taking the slow traffic lane, stopping in designated areas during peak times to reduce congestion, taking alternative routes or car sharing. Such behaviours may also benefit other drivers or groups to which the driver belongs. Part of the challenge within this approach is not only to identify relevant incentives but also gaming techniques that remove some of the need to provide purely financial incentives.

The project will utilize a contextual design [2][3] methodology in order to identify specific incentives and gaming strategies. It is our opinion that the nature of the project requires that we allow the end-users to identify and test the incentives that may work given their particular preferences, circumstances or hard requirements. It is also important to explore the order in which people are given incentives such that we encourage good driving patterns early on while also encouraging them to progress further up the ladder. For example we want to specifically avoid traps where one highergrade incentive is perceived as being worth less than one which is easier to attain.

Motivations are a key part of the driving game that is proposed within I-GEAR, for example we do not envisage that one particular type of incentive will work for all drivers. Instead, through the contextual enquiry process, we will seek to identify combinations of incentives and motivations that are applicable either on an individual 
or group defined basis. Furthermore, we plan to use two main forms of incentive: immediate and status. Immediate rewards will be given when a driver undertakes an action e.g. stopping at a café for which they receive a $1 / 2$ price coffee. Alternatively status rewards or points will be used to encourage longer-term behaviour where drivers collect points for consistently undertaking good actions.

As noted earlier, I-GEAR will also encourage drivers to collaborate and compete through the use of gaming approaches. This will be achieved by offering both individual and social incentives. The underlying main objective always aims to reduce traffic congestion and improve the quality of mobility in Luxembourg.

The originality of our approach is partially reflected by a combination of aspects, all of them potentially raising issues, if they are not properly dealt with, such as:

- Our game concept uses location data and requires users to be identified;

- It is meant to be pervasive as it aims to alter driver behaviour;

- It is meant to share data, enabling collaborative approaches and a shared incentive / reward system;

- It clearly combines real- and virtual-world scenarios and, as such, is a serious game;

- It is meant to integrate an environment of potential high risk, with complex human factors aspects.

\section{$3 \quad$ E-P-T Issues}

We are aware our approach sets several challenges in terms of ethics, privacy and trust. There are other challenges in addition, that will not be dealt with in the context of this paper (e.g. technical aspects, human factors and user acceptance). In the following section, we will discuss separately issues concerning ethics, privacy and to some extent also trust.

\subsection{Ethics Issues}

Ethics requirements and constraints are very strong in social sciences; this also applies to research contexts. [4][5]. The main ethical principles are [4]:

1. Respect for the person's rights and dignity

2. Competence

3. Responsibility

4. Integrity

The first principle strongly relates to legal aspects and aims at a person's privacy, confidentiality, self-determination, autonomy and consistency with the law [5].

The second principle describes how techniques of dealing with patients or conducting research need to meet the highest professional standards of current knowledge and training. 
The third principle refers to scientific responsibilities regarding consequences of one's actions and work. It basically stipulates that a professional way of acting requires you to be accountable for your actions and the ways they are conducted.

The final principle describes how attitudes and actions should be in line with honesty, fairness and respect of others.

While some of these principles may rather obviously link to our use case, some others reflect the fact that they have been set up for defining rules in a therapeutic setup, typically how psychologists should deal with patients. There are other ethical frameworks that more explicitly fit a context of research; the best known and most cited with regard to research in social sciences is the APA code of conduct "Ethical principles of psychologists and code of conduct" [4]. The most relevant principle with regard to our context is that of informed consent. It should be noted that it strongly relates to the first principle described in EFPA. This principle defines that participants have to be thoroughly informed beforehand about the precise processes they are going to face, their outcomes and objectives. In addition, it stipulates that, once the participants have understood all these facets, they are required to express their consent or disapproval, free of any influences.

Relating the principle of informed consent with respect to our game concept, there are two sequential stages that need to be differentiated: the research stage with intermittent experimental setups (simulator in laboratory environment, later on the road) and the final game release. While ethics is quite well defined for research contexts [], we think this is less obvious for final game releases as planned within I-Gear. A crucial point that needs to be dealt with is the end user license agreement (EULA): this allows making sure (1) the user explicitly consents with all the information about the game provided and (2) certifies being of sufficient age. On the other hand, while we think the EULA is a crucial point, we are also convinced it is a very difficult challenge to constitute an EULA that is both sufficiently detailed with regard to information completeness and still sufficiently readable in order to improve chances the user really considered all the information before expressing his consent. Who has not yet been annoyed by "excessively long" EULA texts, scrolling through endless pages, looking for an "OK" button to hit? Of course, bridging between both aspects, explicitness and readability, may constitute an arguable compromise and legal requirements may conflict with those of readability and event ethics to some extent.

Finally, the ethics aspects could also be discussed under a perspective of freedom of choice (e.g. is a pervasive game, namely that developed in I-GEAR, impeding on the freedom of choice?). We think that this question is already covered by our discussion on informed consent und thus will not be treated as a separate question any further here.

\subsection{Privacy Issues}

While ethics requirements with regard to privacy play an important role in social science, they are equally codified in legal provisions. When collecting and analysing different kinds of information in the framework of the I-GEAR project, it is clear that there are legal implications. Questions arising in relation to the amount of data 
collected, the possibilities to share the data with third parties or the general legitimacy of data processing for research purposes are worthy of consideration.

As mentioned before, the I-GEAR project collects different types of information, amongst other, data related to the location of the smart phone, telephone or tablet, GPS data, user IDs, data related to the profile of the user (age, gender, car type, transport references etc.) and data derived from the location information (location data, which direction the users took, preferences regarding the driving behaviour etc.). However, before discussing how to process these data, it is important to distinguish between information and personal data collected during the project. Only when personal data is concerned, some legal limits, named in the following, are applicable. Personal data can be described as "any information relating to an identified or identifiable natural person; an identifiable person is one who can be identified, directly or indirectly, in particular by reference to an identification number or to one or more factors specific to his physical, physiological, mental, economic, cultural or social identity".[6] According to this definition of the EU Data Protection Directive 95/46/EC, many of the data collected during the I-GEAR project meet this definition and are therefore personal data. They are protected by the above mentioned Directive 95/46/EC and by various legal acts transposing this directive into the national law of the EU Member States.

With regard to the standards of processing, it is central that the purpose of the processing must be clearly defined before the processing, which should exclude both the processing for unspecified and unknown purposes and the possibility to subsequently change the original purpose[7]. In more concrete terms this means that the data collected in the framework of the I-GEAR project can only be used for the (predefined) purposes of the project and are not allowed to be used for other, not predefined purposes afterwards. Further, personal data must be "processed fairly and lawfully, collected for specified, explicit and legitimate purposes and not further processed in a way incompatible with those purposes; adequate, relevant and not excessive in relation to the purposes for which they are collected and/or further processed; accurate and, where necessary, kept up to date"[7].

Some other criteria for making data processing legitimate are further detailed in Directive 95/46/EC. One very important condition in the framework of the I-GEAR project is that the processing is legitimate, if the data subject has unambiguously given his consent[8]. The data subject's consent means "any freely given specific and informed indication of his wishes by which the data subject signifies his agreement to personal data relating to him being processed"[9].

Another element of a fair processing of data is the information provided to the data subject. Knowing that one's personal data are processed guarantees transparency and enables the person concerned to assess its own position and to adapt their behaviour to a given situation. The information includes (a) the identity of the controller and of his representative, (b) the purposes of the processing for which the data are intended and (c) any further information in so far as such further information is necessary having regard to the specific circumstances in which the data are collected and to guarantee fair processing in respect of the data subject[10]. 
On condition that the data are collected with respect to the above principles, the question of further use of the data arises. It is planned to share the data originally only used for research purposes with local business partners to ensure that the incentives (e.g. coffee for $1 / 2$ of the price) are used and that the rewards are distributed. While taking into account that this change in purpose has to be communicated to the users from the beginning on and that consent is needed for such use, certain other conditions apply as well. Transferring the data to companies within the EU, which are also subject to the rules of Directive 95/46/EC, has to comply with national law provisions transposing the directive. Transfer to companies outside of the EU is regulated more strictly and requires more protection ${ }^{1}$.

When however discussing the rules of Directive 95/46/EC, it should be taken into account that this instrument will be replaced by a new regulation in $2014^{2}$. This means that EU data protection law will be more effectively harmonised than before: if adopted, the regulation would apply directly in the EU Member States and its provisions would be directly binding. One important change would, for instance, relate to the fact that in case of data breaches, the responsible data protection authority as well as the individual would have to be notified[11].

\subsection{Trust Issues}

Trust issues are the least concrete to deal with and there are multiple valid ways of looking at trust. In contrast to ethics and privacy, it is more of a perceived quality than a tangible and easily controllable quality. In the context of I-GEAR, we think trust is mainly relying on two requirements:

1. Strict compliance with and transparency of that compliance with both ethics and legal aspects;

2. Specific emphasis on those principles that reassure a user our pervasive game is developed in a way that it will not jeopardize driving safety under any condition, provided the user behaves as a responsible driver;

While the first requirement may easily be generalized over a broader context, the second one is much more specific to high-risk domains. In addition, there remain questions about the trustworthiness of a system that intents to alter your driving behaviour: some people may perceive this property of a system as not acceptable and as a result not be in favour of pervasive games. This of course assumes they understand the term as a precise definition, especially when used in the public context this may not be immediately obvious. Finally, on a less general level, there may also be doubts regarding the performance of a system or game that has an impact on your driving waypoints: does this system suggest the best route in regard of the user's preferences

\footnotetext{
${ }^{1}$ For details, compare Article 25 and 26 of the Directive 95/46/EC, OJ 1995, L-281/31.

${ }^{2}$ Proposal for a Regulation of the European Parliament and of the Council on the protection of individuals with regard to the processing of personal data and on the free movement of such data (General Data Protection Regulation), 25 January 2012, COM (2012) 11 final.
} 
and the system's expected behaviour? This applies to many properties that define a navigation path: travel time, travel distance, fuel economy, etc.

It appears, trust is a very specific property and, as a perceived property, may be extremely dependent not only on the system itself, but very much on the users' specific expectations. We think I-GEAR has to consider the two above-mentioned requirements and also look more specifically into the results on user acceptance testing in order to understand whether there may be issues of trustworthiness amongst those cases where user acceptance is not given.

\section{Conclusion}

While not all aspects of ethics, privacy and trust could be discussed in this paper, we believe that the brief discussion presented should inform people about these aspects with respect to serious gaming. At present it is our view that these issues are at times not sufficiently explored, and indeed this is due in part to the contradiction which arises between aspects such as informed consent and the basics of playing serious games. In other respects it is simply that such issues have not been considered in the context of serious gaming merely due to its often perceived fringe appeal or level of interest. Furthermore, another explanation could be that gathering the interdisciplinary expertise is a difficult challenge in a domain that often concentrates on technical aspects. Our work within this area is still evolving and we expect to publish more about our experiences within I-GEAR at a later date.

Regarding trust issues, we highlighted how we think trust is linked to both ethics and privacy within a conditional relationship while an important part of trust also falls into the domain of user acceptance. We explained why we think that ad-hoc approaches only work to some extent when it comes to guaranteeing trust; in contrast to ethics and privacy, there is no guarantee that respecting predefined requirements is sufficient for establishing trustworthiness.

Finally, it is interesting to note that most of the requirements regarding ethics and privacy call for the same underlying principles that are expressed in different terms while converging towards the same objective of protecting a person's rights.

Acknowledgements. The I-GEAR project is partially funded by Fonds National de la Recerche, Luxembourg under grant number 11/IS/1204159. The authors would also like to acknowledge other members of the I-GEAR project including: Thomas Engel, Nicolas Louveton, Tigran Avanesov and Martin Kracheel.

\section{References}

1. Bliemer, M.C.J., van Amelsfort, D.H.: Rewarding instead of charging road users: a model case study investigating effects on traffic conditions. In: Proceedings of the third Kuhmo-Nectar Conference (2008)

2. Beyer, H., Holtzblatt, K.: Contextual design: designing customer-centered systems. Morgan Kaufmann Pub. (1998) 
3. Holtzblatt, K.: Rapid Contextual Design: A How-to Guide to Key Techniques for UserCentered Design (Interactive Technologies), p.324. Morgan Kaufmann (2004)

4. The Official EFPA meta-code of ethics, http://www. efpa. eu/ethics

5. The Official APA Ethics Code, http: / /www . apa.org/ethics/index.aspx

6. Article 2 a of the EU Data Protection Directive 95/46/EC, OJ 1995, L-281/31

7. Article 6 of the EU Data Protection Directive 95/46/EC, OJ 1995, L-281/31

8. Article 7 a of the EU Data Protection Directive 95/46/EC, OJ 1995, L-281/31

9. Article $2 \mathrm{~h}$ of the EU Data Protection Directive 95/46/EC, OJ 1995, L-281/31

10. Articles 10 and 11 of the Directive 95/46/EC, OJ 1995, L-281/31

11. Articles 31 and 32 Proposal for a General Data Protection Regulation, 25 January 2012, COM (2012) 11 final 